Original Article

\title{
The Gross Total Resection and Molecular Markers in Grade II Glioma
}

\author{
ljaz Hussain Wadd, Shahid Mukhtar, Syed Arslan Haider, Sidra ljaz, Naeem-ul-Hassan, \\ Muhammad Anwar Chaudhary \\ Department of Neurosurgery, Punjab Institute of Neurosciences (PINS), Lahore - Pakistan
}

\section{ABSTRACT}

Objective: The study determined the importance of gross total resection in grade II Glioma and evaluated the importance of tumor markers as prognostic factors.

Material and Methods: We included the 240 patients aged $13-65$ years with supratentorialsuspected lowgrade Glioma. Craniotomy was done in all my patients under general anesthesia and excised the tumor safely with the help of a microscope and CUSA without causing any focal deficit or hemodynamic instability. The 3D conformal radiotherapy and or Temozolomide chemotherapy was started as advised by the oncologist, postoperatively. The 5 and 10 years' overall survival and progression-free survival were evaluated in my study.

Results: Median age of the patients was 45 years. The $46.66 \%$ patients were IDH mutant Astrocytoma, 39.1 $6 \%$ patients were IDH mutant Oligodendroglioma with loss of heterozygosity at I p/19q levels, and $14.16 \%$ patients had IDH wild type Astrocytoma. The gross total resection was done in 113 patients, subtotal in 53 patients, partial resection in 45 patients, and biopsy in 29 patients. Postoperative radiotherapy was done in 170 patients and Temozolomide chemotherapy in 67 patients. The 5 and 10 years' progression-free survival was $80 \%$ and $49 \%$ and overall survival was $86.3 \%$ and $67 \%$. The 10 - year overall survival for Oligodendroglioma, the IDH mutant Astrocytoma, and IDH wild Astrocytoma were $93 \%$, 61.6\%, and 34.7\% (respectively), and progression-free survival were $89.2 \%, 48 \%$, and $34 \%$ (respectively). The histologically proven Oligodendroglioma with gross total excision and chemotherapy and radiotherapy had no recurrence.

Conclusion: The gross total resection of IDH mutant Astrocytoma had a good outcome.

Keywords: Gross Total Excision, Subtotal Excision, IDH (Isocitrate Dehydrogenase), Overall Survival, Progression-Free Survival.

Corresponding Author: ljaz Hussain Wadd

Department of Neurosurgery, Punjab Institute of

Neurosciences (PINS), Lahore - Pakistan

Email:drejazns@gmail.com

Date of Submission: 07-08-2021

Date of Revision: 09-09-2021

Date of Revision: 15-09-2021

Date of Online Publishing: 30-9-2021

Date of Print: 30-9-2021

\section{INTRODUCTION}

Many tumors can originate intra-cranially and include glioma, meningioma, neuroma, adenoma, etc. $75 \%$ of tumors of the brains are glioma. ${ }^{1}$ Mostly gliomas are in supra-tent but they can be infra-tent and in the spinal cord. They are common in the frontal lobes of the brain followed

DOI: 10.36552/pjns.v25i3.599 
by the temporal lobes. They have infiltrative growth of glial cells and spreads along white matter tracks. They are classified as Astrocytoma, Oligodendroglioma, or mixed Glioma. WHO in 2016 gives their classification as grades 11, III, and IV. ${ }^{2}$ Glioblastoma multiform is grade IV and highly malignant tumors. ${ }^{1-3}$ The exact etiology of brain tumors is not known but different risk factors of gliomas are radiation exposure in pediatric age 3 and the genetic factor. ${ }^{4}$ WHO classified grade II Oligodendroglioma, the astrocytoma, and the mixed tumors Oligoastrocytoma as low grade. ${ }^{5}$ LGG has slow growth rate with no signs of high intracranial pressure and most commonly presents with fits and had a good outcome after good excisions. ${ }^{6}$ updated WHO classification of 2016 also gives stress on molecular markers like IDH (Isocitrate Dehydrogenase) gene mutation and chromosome I $\mathrm{p} / \mathrm{I} \mathrm{qq}$ codeletion for tumors prognosis. ${ }^{7}$ The studies available in the literature about the prognosis of low-grade Glioma are on the old classification of Gliomas and very few studies are available to determine the importance of subtypes based on molecular markers as a prognostic factor of low-grade Glioma. Uptill now the standard treatment available for Glioma is maximum possible resection followed by radiotherapy and chemotherapy or Temozolomide. In less than 40 years old patients with subtotal excision and low-grade Glioma, it is still controversial how to plan treatment concerning molecular markers with no universal consensus on it. So we conducted the study to determine the importance of these tumors markers and their impact on the prognosis of the patients.

\section{MATERIAL AND METHODS}

\section{Study Setting}

The study included 240 patients of histologically proven low-grade Glioma operated in Neurosurgery Unit 2 from January 2010 to January 2018. The informed consent was taken from all patients. Ethical approval was taken from the ethical committee.

\section{Inclusion Criteria}

All the patients of age 13-65 years of age with suspected low-grade Glioma on MRI were included in the study.

\section{Exclusion Criteria}

The patients more than 65 years of age, medically unfit, posterior cranial fossa Glioma, brainstem, spinal cord, and high-grade Gliomas were excluded from our study.

\section{Surgical Management and Resections}

We did craniotomy in all our patients under general anesthesia and excised the tumor with the help of a microscope and CUSA without causing any neurological deficit or hemodynamic instability. After the surgery, ICU care was given to all our patients and the patient shifted to the ward after 24hours. After 24 hours MRI brain plane and contrast were obtained.

\section{The Extent of Resections (EORs)}

The extent ofresection of the tumor was classified with the help of MRI and operation notes as:

1. Gross total (GTR) if resection more than $99 \%$, 90 - 99\%.

2. Removal as subtotal.

\section{Patient Resection}

3. $50-90 \%$ as partial resection (PR).

4. Less than $50 \%$ removal was a biopsy. After the histopathological report, we further studied the molecular markers. 


\section{Clinical Management}

All patients were treated in the ward and discharged on the 6th postoperative day when the wound is healed. Then patients were referred to an oncologist for 3D conformal radiotherapy and/or chemotherapy.

\section{Progression Free Survival}

The progression free-survival was measured from the date we received the biopsy report to the date we received any report of MRI as recurrence of tumor growth and overall survival as the date from biopsy report till patient breath it's last. The progression free-survival was measured from the date we received the biopsy report to the date we received any report of MRI as recurrence of tumor growth.

\section{Overall Survival}

As the date from biopsy report till patient breath it's last.

\section{Data Analysis}

All the data was collected and entered on a proforma. The SPSS version 25.0 was used for all statistical analyses.

\section{RESULTS}

\section{Age Distribution}

In our study, patients of $13-65$ years of age were studied with a median age of patients 45 years.

\section{Gender Distribution}

Among 240 patients, there were 120 (50\%) males and 120 (50\%) females.

The $94 \quad(39.16 \%)$ patients were Oligodendroglioma with I p/l9q co-deleted, 112 (66\%) had 1DH mutant Astrocytoma and 34 (14.16\%) had IDH wild type Astrocytoma.

\section{Information on EORs, Tumor, and Drugs}

The gross total resection was done in 113 (47.08\%) patients, subtotal resection in 47 (19.58\%) patients, partial resection in 67 (27.91\%), and biopsy in 13 (5.41\%). In 137 (57.08\%) patients, the tumor was located in the frontal lobes of the brain. The 135 (56.25\%) patients received 3D conformal radiotherapy, postoperatively. The 73 (30.40\%) received PCV/ Temozolomide chemotherapy and 54 (22.50\%) patients received both radiotherapy and chemotherapy. The Oligodendroglioma was mostly in young patients and frontal lobes location and IDH wild type astrocytoma in nonfrontal lobe location (Table 1).

\section{Survivals, Treatment, and Follow-up}

All the patients were followed up for 60 months. The treatment failure was defined as recurrent tumor visible on postoperative MRI or progressive increase in the size of the tumor. The treatment failure was seen in 72 (30\%) patients during follow-up; 16 (17.02\%) Oligodendroglioma, 37 (33.03\%) in IDH mutant type of astrocytoma, and 19 (55.88\%) IDH wild type of Astrocytoma. In Oligodendroglioma,the median treatment failure time was 53.7 months and for IDH mutant variant of Astrocytoma 38 months, and IDH wild variant of Astrocytoma was 18.3 months. The tumor progression-free survival rate was $80 \%$ and $49 \%$ and overall survival was $86.3 \%$ and $67 \%$ for 5 and I0 years, respectively. The overall survival rate was $93 \%, 61.6 \%$, and $34.7 \%$ (respectively) and progression-free survivalrates were $89.2 \%$, 48\%, and $34 \%$ (respectively) for 10 years respectively for Oligodendroglioma, IDH mutant, and wildtype Astrocytoma. The other variable showed that gross total removal, IDH mutant variant, and $\mathrm{Ip} / \mathrm{I9q}$ co-deletion, and $4 \mathrm{~cm}$ size of tumor were good prognostic factors (Table 2). 


\begin{tabular}{|c|c|c|c|c|c|}
\hline Variable & Level & N (\%) & $\begin{array}{l}\text { Oligodendroglioma } \\
\qquad(N=94)\end{array}$ & $\begin{array}{c}\text { IDH Mutant } \\
\text { Astrocytoma } \\
(\mathrm{N}=112)\end{array}$ & $\begin{array}{c}\text { IDH Wild } \\
\text { Astrocytoma } \\
(\mathbf{N}=34)\end{array}$ \\
\hline \multirow{2}{*}{ Age } & $<40$ years & $110(45.83)$ & $28(29.87)$ & 59 (52.67) & $23(67.64)$ \\
\hline & $\geq 40$ years & $130(54.16)$ & $66(70.21)$ & 53 (47.32) & $11(32.35)$ \\
\hline \multirow{2}{*}{ Sex } & $M$ & $120(50)$ & 27 (28.72) & $70(62.50)$ & $23(94.11)$ \\
\hline & $\mathrm{F}$ & $120(50)$ & 67 (71.27) & $42(37.50)$ & $11(32.35)$ \\
\hline \multirow{2}{*}{ Size of tumor } & $<4 \mathrm{~cm}$ & 165 (68.75) & 67 (71.27) & 77 (68.75) & $21(61.76)$ \\
\hline & $\geq 4 \mathrm{~cm}$ & 75 (31.25) & 27 (28.72) & 35 (31.25) & 13 (38.25) \\
\hline \multirow{2}{*}{ Resection } & Gross total & $113(47.08)$ & $53(56.38)$ & $40(35.71)$ & $20(58.82)$ \\
\hline & Non-GTR & 127 (52.91) & $41(43.61)$ & $72(64.28)$ & $14(41.17)$ \\
\hline
\end{tabular}

Postoperative radiotherapy was not a good prognostic factor but chemotherapy shows a good prognosis in molecular subtypes of IDH mutant variant of Astrocytoma and with I $\mathrm{p} / \mathrm{I} 9 \mathrm{q}$ codeletion.
Table 2: 10 years' overall survival and progression-free survival.

\begin{tabular}{lccc|} 
Variable & $\mathbf{N}(\mathbf{\% )}$ & $\begin{array}{c}\text { 10 Year - OS } \\
\mathbf{( 4 9 \% )}\end{array}$ & $\begin{array}{c}\text { 10 Year - PFS } \\
\mathbf{( 6 7 \% )}\end{array}$ \\
< 40 years & $110(45.83)$ & 75.40 & 61.34 \\
$\geq 40$ years & $130(54.16)$ & 58.34 & 41.80 \\
IDH Mutant Astrocytoma. & $112(46.66)$ & 61.60 & 48 \\
Oligodendroglioma. & $94(39.16)$ & 93 & 89.20 \\
IDH wild Astrocytoma. & $34(14.16)$ & 34.7 & 34 \\
< 4 cm size & $165(68.75)$ & 67.73 & 61.33 \\
$\geq 4$ cm size & $75(31.25)$ & 46.88 & 41.21 \\
Gross total resection. & $113(47.08)$ & 80.6 & 66.1 \\
Non-GTR & $127(52.91)$ & 50.9 & 41.3 \\
Chemotherapy. & $93(38.75)$ & 76.6 & 66.2 \\
\hline
\end{tabular}

\section{DISCUSSION}

The main focus in our study was on molecular markers of Glioma whether they are IDH mutant or IDH wild type or there is any I p/I9q codeletion. We proved in our study that IDH mutant and I $p / I 9 p$ codeletion are associated with good prognosis and IDH wild with bad prognosis. WHO classification 2016 also showed the same that molecular markers of the tumors have a strong correlation with prognosis and over-rule the histological diagnosis. ${ }^{8}$ Second thing that we studied was the extent of resection of the tumor and its correlation with overall survival and progression-free survival. We concluded in our study that gross total resection is directly related to good overall survival and progression-free survival than non-gross total resection. We did the maximum safe resection in all molecular subtypes of Glioma. Many other studies are available that suggest that early surgery with gross total resection has good overall survival and progression-free survival in low-grade Glioma. The study by Cordier et al. showed that volumetric measurements and extent of resection are independent prognostic factors in addition to molecular markers. ${ }^{9}$ Smith et al. ${ }^{10}$ also concluded in their study that a larger extent of tumor excision is directly related to the longer survival of the patients and with small residual tumor had a major impact on overall survival of the patients. A recent paper by Wahl et al $^{11}$ proved that postoperative residual tumor has an impact on overall survival and correlated it with molecular subtype. We tried to do maximum excision of the tumors and in our study gross total excision was done in II3 (47.08\%) patients. In another study 
they did gross total resection safely in $4-15 \%$ which is lowerthan our study. ${ }^{13}$ We achieved relatively high overall survival and progressionfree survival thanother studies. Another study also proves that the extent of surgical resection of Glioma has a great role inthe prognosis of the patients. ${ }^{12}$ We alsoobserved the extent of tumor excision is directly related to recurrence of the tumor and overall survivals of the patients. No redo surgery was done for any residual tumor on post-op MRI. Inanother study, they also assessed whether the tumor is removed completely or any residual by early MRI orCT scan brain plane and contrast and diffusion-weighted images in 24 72 hours of operation. ${ }^{14}$ Werefer all ourpatients toan oncologist for radiotherapy and PCV chemotherapy or Temozolomide. We have proved in our study that radiotherapy does not affect the overall survival andprogression-free survival and high dose radiotherapy has its side effects too. But we observed in the study that 12 cycles of Temozolomide chemotherapy increase the overall survival and progression-free survival in IDH mutant and Ip/I9q codeletion positive patients. In other studies they also used Temozolomide either in recurrence ${ }^{15}$ or in freshly diagnosed patients. ${ }^{16}$ In these studies when they used $6-12$ cycles of 28 days each at the dosage of $200 \mathrm{mg} / \mathrm{m}^{2}$ per day for 5 days every cycle they have seen a partialresponse rate of $10 \%$ to $47 \%$. The 12 month progression-free survival rate was $39 \%$ in one series ${ }^{17}$ and $73 \%$ to $75 \%$ in other series. ${ }^{15}$ Jenkins et al $^{18}$ also showed in series that that newly diagnosed Glioma with loss of heterozygosity at I $p / 19 q$ had a better median survival of I 1.9 years than 8.1 years in those Glioma caseswithout loss of heterozygosity at Ip/19q.

\section{CONCLUSION}

From our study we concluded that:

1. The histologically proven Oligodendroglioma with gross total excision and chemotherapy and radiotherapy had no recurrence.

2. The gross total resection of IDH mutant astrocytoma had a good outcome.

\section{Limitation of the Study}

It was a single-center study with a small no of patients and patients gets oncological care from different centers and different oncologists.

\section{REFERENCES}

1. Ostrom QT, Gittleman $\mathrm{H}, \mathrm{Xu}$ J, et al. CBTRUS statistical report: primary brain and other central nervous system tumors diagnosed in the United States in 2009-2013. Neuro Oncol. 2016; 18 (Suppl_5): v1-v75.

2. Perry $A$, Wesseling P. Histologic classification of gliomas. HandbClin Neurol. 2016; 134: 71-95.

3. Preston DL, Ron E, Yonehara S, et al. Tumors of the nervous system and pituitary gland associated with atomic bomb radiation exposure. J Natl Cancer Inst. 2002; 94 (20): 1555-1563.

4. Wrensch $M$, Jenkins RB, Chang JS, et al. Variants in the CDKN2B and RTEL1 regions are associated with high-grade glioma susceptibility. Nat Genet. 2009; 41 (8): 905-908.

5. Louis DN, et al. The 2007 WHO classification of tumours of the central nervous system. Actaneuropathologica. 2007; 114: 97-109.

6. Claus EB, et al. Survival and low-grade glioma: the emergence of genetic information. Neurosurgical Focus, 2015; 38: E6.

Doi: 10.3171/2014.10.FOCUS12367.

7. Forst DA, Nahed BV, Loeffler JS, Batchelor TT. Lowgrade gliomas. The Oncologist, 2014; 19: 403-413. Doi: 10.1634/theoncologist.2013-0345.

8. Louis DN, Perry A, Reifenberger G et al. The 2016 World Health Organization classification of tumors of the central nervous system: a summary. Acta Neuropathol. 2016; 131 (6): 803-820.

9. Cordier D, Gozé C, Schädelin S, Rigau V, Mariani L, Duffau $\mathrm{H}$. A better surgical resectability of WHO grade II gliomas is independent of favorable molecular markers. J Neurooncol. 2015; 121 (1): 185-193.

10. Smith JS, Chang EF, Lamborn KR et al. Role of 
extent of resection in the long-term outcome of low-grade hemispheric gliomas. J Clin Oncol. 2008; 26 (8): 1338-1345.

11. Wahl $M$, Phillips JJ, Molinaro AM et al. Chemotherapy for adult low-grade gliomas: clinical outcomes by molecular subtype in a phase II study of adjuvant temozolomide. Neuro Oncol. 2017; 19 (2): 242-251.

12. Pessina, F. et al. Value of Surgical Resection in Patients with Newly Diagnosed Grade III Glioma Treated in a Multimodal Approach: Surgery, Chemotherapy and Radiotherapy. Ann Surg Oncol. 23, 3040-3046 (2016).

13. Wijnenga MMJ, et al. The impact of surgery in molecularly defined low-grade glioma: an integrated clinical, radiological, and molecular analysis. Neuro-oncology, 2018; 20: 103-112.

Doi: 10.1093/neuonc/nox176.

14. Vogelbaum MA, Jost $S$, Aghi MK, et al. Application of novel response/progression measures for surgically delivered therapies for gliomas:
Response Assessment in Neuro-Oncology (RANO) Working Group. Neurosurgery, 2012; 70 (1): 23443; Discussion 43-4.

15. Tosoni A, Franceschi $E$, Ermani $M$, et al. Temozolomide three weeks on and one week off as first line therapy for patients with recurrent or progressive low grade gliomas. J Neurooncol. 2008; 89: 179-185.

16. Kesari S, Schiff D, Drappatz J, et al. Phase II study of protracted daily temozolomide for low-grade gliomas in adults. Clin Cancer Res. 2009; 15: 330337.

17. Pace A, Vidiri A, Galiè E, et al. Temozolomide chemotherapy for progressive low-grade glioma: Clinical benefits and radiological response. Ann Oncol. 2003; 14: 1722-1726.

18. Jenkins RB, Blair $H$, Ballman $K V$, et al. $A$ $t(1 ; 19)(q 10 ; p 10)$ mediates the combined deletions of $1 p$ and $19 q$ and predicts a better prognosis of patients with oligodendroglioma. Cancer Res. 2006; 66: 9852-9861.

\section{Additional Information}

Disclosures: Authors report no conflict of interest.

Ethical Review Board Approval: The study was conformed to the ethical review board requirements.

Human Subjects: Consent was obtained by all patients/participants in this study.

\section{Conflicts of Interest:}

In compliance with the ICMJE uniform disclosure form, all authors declare the following:

Financial Relationships: All authors have declared that they have no financial relationships at present or within the previous three years with any organizations that might have an interest in the submitted work.

Other Relationships: All authors have declared that there are no other relationships or activities that could appear to have influenced the submitted work.

\section{AUTHORS CONTRIBUTIONS}

\begin{tabular}{|l|l|l|}
\hline Sr.\# & Author's Full Name & Intellectual Contribution to Paper in Terms of: \\
\hline 1. & ljaz Hussain Wadd & Study Design and Methodology. \\
\hline 2. & Shahid Mukhtair & Paper Writing and Data Calculations. \\
\hline 3. & Syed Arslan Haider & Data Collection and Calculations. \\
\hline 4. & $\begin{array}{l}\text { Sidra ljaz, Naeem ul } \\
\text { Hassan }\end{array}$ & Analysis of Data and Interpretation of Results etc. \\
\hline 5. & Anwar Chaudhry & Literature Review and Referencing. \\
\hline
\end{tabular}

